\title{
BMJ Open Early childhood vaccination and subsequent mortality or morbidity: are observational studies hampered by residual confounding? A Danish register-based cohort study
}

\author{
Andreas Jensen, ${ }^{\oplus 1}$ Per Kragh Andersen, ${ }^{2}$ Lone Graff Stensballe ${ }^{\oplus 1}$
}

To cite: Jensen A, Andersen PK, Stensballe LG. Early childhood vaccination and subsequent mortality or morbidity: are observational studies hampered by residual confounding? A Danish register-based cohort study. BMJ Open 2019;9:e029794. doi:10.1136/ bmjopen-2019-029794

- Prepublication history and additional material for this paper are available online. To view these files, please visit the journal online (http://dx.doi. org/10.1136/bmjopen-2019029794).

Received 12 February 2019 Revised 06 August 2019 Accepted 29 August 2019
Check for updates

(C) Author(s) (or their employer(s)) 2019. Re-use permitted under CC BY-NC. No commercial re-use. See rights and permissions. Published by BMJ.

${ }^{1}$ The Child and Adolescent Clinic Copenhagen University Hospital, Copenhagen, Denmark

${ }^{2}$ Section of Biostatistics, Department of Public Health, University of Copenhagen, Copenhagen, Denmark

Correspondence to Andreas Jensen; andreas.jensen.01@regionh.dk

\section{ABSTRACT}

Objectives To estimate the association between childhood vaccination and subsequent morbidity and mortality by adjusting for environmental and host factors. Further, to examine the degree of residual confounding in such observational studies.

Design Register-based cohort study including 1122929 Danish children.

Participants All children born in Denmark in the period 1999-2016 who survived until 16 months of age without prior migration followed from 16 months until the first of the following: event of interest, migration, 5 years of age or 31 December 2016.

Main outcome measures Adjusted HRs (aHRs) and absolute risks were calculated for the three outcomes: mortality, hospitalisation for infection and asthma using register data on deaths, specific hospital contacts and dispensed prescribed medication. The exposure was the combination of the routine vaccines against diphteriatetanus-pertussis-polio-Haemophilus influenzae type b and measles-mumps-rubella (DTP and MMR in short) administered in early childhood. Hospitalisation due to accidents was analysed as a negative control outcome to examine residual confounding.

Results Children with 3DTP+MMR had a lower hazard of mortality than the reference group with 3DTP, adjusted HR $(\mathrm{aHR})=0.45$ (95\% Cl: 0.35 to 0.57$)$, whereas the children with 1 or 2 DTP had higher hazards of dying, aHR=1.55 (95\% Cl: 1.14 to 2.13 ) and aHR=1.96 (95\% Cl: 1.34 to 2.89). The vaccination group $3 D T P+M M R$ was associated with a reduced hazard of asthma aHR $=0.94(95 \% \mathrm{Cl}: 0.92$ to 0.96 ). Also, the vaccination group 3DTP+MMR was associated with a reduced hazard of hospitalisation due to accidents, aHR=0.83 ( 0.80 to 0.85$)$ compared with the reference group with 3 DTP.

Conclusions The results suggested a beneficial impact of MMR on under-five mortality but did not support the hypothesis that DTP is detrimental, since the group of children with fewer DTP vaccinations experienced increased mortality. The results of the study may to some degree be prone to residual confounding since an unexpected association between MMR vaccination and hospitalisation for accidents was observed.
Strengths and limitations of this study

Large sample size.

- National cohort study design.

- Estimation of absolute risks.

- Observational study design.

> Possibility of unmeasured confounding.

\section{INTRODUCTION}

Studies suggest that some of the vaccines routinely administered in early childhood may affect the risk of illness and death from conditions other than the targeted infectious diseases they are designed to prevent. The overall hypothesis about the so-called non-specific effects of vaccines is that live-attenuated vaccines such as measles-containing vaccines may lower subsequent risk, whereas subunit vaccines such as the diphtheriatetanus-pertussis-polio-Haemophilus influenzae type b (DTP) vaccine may increase risk of illness and death from other causes than the vaccine target disease. ${ }^{12}$

The beneficial non-specific effects of the measles-containing vaccine measles-mumpsrubella (MMR) were indicated in three Danish observational studies. ${ }^{3-5}$ A study found an increased risk of hospitalisation for lower airway infection in the small group of children who received DTP and MMR simultaneously. ${ }^{6}$ Potential beneficial effects might not be restricted to measles-containing vaccines. A recent Dutch study found a protective effect of receiving further DTP-like vaccines on the risk of hospitalisation for infection. ${ }^{7}$

However, observational vaccine studies can be prone to 'healthy vaccinee bias'. Healthy children are more likely to be vaccinated so the 'effects' of vaccination may be explained by general better health in vaccine 
recipients. ${ }^{8-11}$ The ability to receive the next vaccine in the child vaccination programme indicates good health, and it might not matter whether this vaccine is inactivated (DTP-like) or live-attenuated (MMR-like). Further, the clinical indication for the two vaccine types are different: live vaccines as measles-containing vaccines are contraindicated in severely immunosuppressed individuals since (despite attenuation) the vaccine may replicate and infect the recipient. ${ }^{12-14}$ Individuals who also receive live vaccines are probably more immunocompetent than those who only receive subunit vaccines. Hence, vaccination status may be a result of good health and not a cause. This bias is difficult to accommodate, ${ }^{15}$ so potential non-specific effects of vaccines are ideally tested in randomised trials. In a recent large randomised trial, we studied the potential (a priori thought to be beneficial) non-specific effect of another live-attenuated vaccine, Bacillus Calmette-Guérin (BCG), on early childhood hospitalisation in Denmark, but no non-specific effect was found. ${ }^{16}$

In the present national cohort study, we examine determinants of exposure to all combinations of the MMR and DTP vaccines which are scheduled in early childhood in the Danish vaccination programme. Since such host and environmental factors are likely to confound the relationship between vaccination exposure and subsequent morbidity and mortality, we present the results of adjusted analyses. We defined a detailed exposure capturing all combinations of DTP and MMR.

\section{METHODS}

The cohort consists of all children liveborn in Denmark in the period 1999-2016 who survived until 16 months of age without prior migration. They were all followed from 16 months until the first of the following events: the event of interest, death, registered migration out of Denmark, 5 years of age or 31 December 2016.

\section{Patient and public involvement}

The study was based on pseudonymised national registry data. Hence no patient and public involvement was required. In this register-based study, we had no direct patient contact, and no human subjects review board was required. This is the usual procedure for register-based studies. Data in the public health registers are available to researchers on request and subject to permission from the authorities.

\section{Registries}

The study was based on the Danish health registers ${ }^{17}$ as well as registers from Statistics Denmark ${ }^{18}$ with information on mortality and socioeconomic variables. The Central Person Registration (CPR) ${ }^{19}$ enabled linking of data on an individual level. The following health registers were used: The National Patient Register ${ }^{20}$ The Register of Medicinal Product Statistics, ${ }^{21}$ The Medical Birth Registry ${ }^{22}$ and The Danish National Health Service
Register (NHSR) ${ }^{23}$ from which the vaccine information was obtained. For more details, consult online supplementary table S1.

\section{Vaccination data}

The vaccines in the first 15 months of the Danish child vaccination programme ${ }^{24}$ include three administrations of the inactivated vaccines, the pentavalent DTP 1 at 3, 5 and 12 months of age, and one administration of the live-attenuated MMR at 15 months of age (see online supplementary table S2). We refer to the vaccines as DTP and MMR. The DTP used in Denmark is acellular (subunit vaccine).

The vaccine information in the NHSR was based on invoices from general practitioners. The invoices are necessary for the general practitioner to obtain reimbursement. This information is collected in NHSR where data can be extracted using health service codes of, for example, the relevant vaccinations. A complete list of the health service codes used in the present study can be found in the online supplementary table S3.

NHSR registers services on a weekly basis. We defined the vaccinations to take place on the Wednesday of the corresponding week. Multiple administrations of the same vaccine within the same week were considered as one vaccination.

\section{Exposure definition: vaccination status}

Vaccination status was defined as a time-dependent exposure with the following eight values:

1. 1DTP: One DTP, no MMR

2. 2DTP: Two DTP, no MMR

3. 3DTP: Three DTP, no MMR

4. 1DTP+MMR: One DTP and MMR

5. 2DTP+MMR: Two DTP and MMR

6. 3DTP+MMR: Three DTP and MMR

7. None: neither any DTP nor MMR

8. MMR: MMR, no DTP

The vaccination status for each child changed following each additional vaccination after 16 months of age until the end of follow-up. The children without any DTP vaccinations in groups 7 and 8 are not of primary interest but are defined and included for completeness.

\section{Outcomes: mortality and morbidity}

Three main outcomes were considered to evaluate the non-specific effects of vaccination status on mortality and morbidity: (1) mortality, (2) hospitalisation for infection and (3) onset of asthma. These outcomes were also used in prior studies of potential non-specific effect of vaccines. ${ }^{4-62526}$ Only time to first hospitalisation for infection was analysed, and the diagnoses used in the definition are stated in the online supplementary table S4. The asthma outcome was defined using a customised version of an already published and validated algorithm for ascertainment of asthma, ${ }^{27} 28$ but where the event used in this paper was defined using only hospitalisations and prescriptions between 16 months and 5 years of age. 


\section{Potential confounders}

Confounders were defined as potential causes of exposure as well as outcome. ${ }^{29}$ Twelve potential confounders indicating general health status were ascertained at 16 months of age for each child, and they were all included in the adjusted analyses. Five continuous confounders were included: number of hospitalisations for infection, number of other hospitalisations, number of dispensed antibiotics prescriptions, number of other dispensed prescriptions and age in days at first discharge to the home after birth. Seven categorical confounders were included: gender (male/female), presence of chronic disease (yes/ no), presence of atopic disease (yes/no), small for gestational age $($ yes $/$ no) $){ }^{30}$ prematurity (gestational age $<37$ weeks), maternal education ( $<10$ years, $10-12$ years, $>12$ years) and ethnicity (Danish/other) defined by Statistics Denmark. ${ }^{18}$ The last two variables were considered socioeconomic proxies, whereas the remaining variables were considered proxies of the host health status.

\section{Definition of children with chronic or atopic disease}

Chronic disease or atopic disease may lead to recurrent infection and, consequently, postponed vaccinations. Children with chronic disease were identified using an algorithm using register data on diagnoses of chronic disease in relation to hospital admissions. ${ }^{31}$ Note that the definition of chronic disease did not include prescriptions. Children with atopic disease were identified in a similar fashion using a validated algorithm, ${ }^{27}{ }^{28}$ but in this case data on prescribed medication use were also taken into account. The disease status was evaluated and fixed at 16 months of age.

\section{Statistical methods}

The analyses involved estimation of the association between vaccination exposure and health status through the main outcomes: mortality, hospitalisation for infection and onset of asthma.

Statistical analyses were performed using Stata/MP 15.1. and $\mathrm{R}$ version 3.5.1 using the riskRegression package. ${ }^{32}$

\section{Association between confounders and vaccination group}

Note that each potential confounder was included in the models irrespective of the magnitude of its marginal association. ${ }^{29}$ Given the large sample size, the risk of overfitting was minimal as the number of confounders was kept relatively low.

\section{Association between vaccination group exposure and outcomes}

The mortality outcome was analysed using Cox regression. The underlying time scale was age with entry at 16 months of age and exit at 5 years of age. Children emigrating out of Denmark before 16 months of age were excluded, and children emigrating between 16 months and 5 years of age were censored at age of emigration. For the categorical variables with missing values, a separate category indicating missingness was defined. For each outcome, the crude HRs were presented along with the HRs obtained after adjustment for the 12 confounders
(aHR). The proportional-hazards assumption was tested using Schoenfeld residuals.

For the two non-mortality outcomes, the cause-specific HRs were estimated using a Cox model. However, absolute and relative risks are easier to interpret than HRs. To estimate absolute risks of experiencing the events of interest (at different landmark time points), the competing event of death was accommodated by fitting separate cause-specific hazards models and then applying the g-formula to the results. ${ }^{33}$ These risks represent average causal effects under the hypothesis of no unmeasured confounders. ${ }^{34}$ The vaccination-specific absolute risks of experiencing the three main outcomes before 5 years of age were estimated from five different landmark times to accommodate the time-dependent nature of vaccination status. The five landmark times were chosen as 16, 20, 24, 36 and 48 months of age. The risk predictions from each landmark analysis were based on a model fitted to the event-free children who survived up to the given landmark age. No smoothing of the estimated coefficients across landmark models was performed. ${ }^{35}$

In all analyses the reference group was chosen as the group of children with 3 DTP, but no MMR. Children with 3 DTPs also represented the reference group in prior Danish studies of non-specific effects of vaccination.

Finally, the potential presence of residual confounding was assessed by analysing hospitalisation for accidents (injuries) as a negative control outcome. ${ }^{36}$ These types of hospitalisations are not expected to be related to previous vaccination history.

\section{RESULTS}

\section{Distribution of vaccination groups}

Table 1 shows that the eight different vaccination groups defined in the study varied considerably in size. For example, children with neither DTP nor MMR at 16 months of age represented 18761 children constituting only $1.67 \%$ of the children in the cohort. On the other

Table 1 Absolute and relative numbers of children in the study by vaccination status at baseline (16 months of age)

\begin{tabular}{lc}
\hline Vaccination status & No of children (\%) \\
\hline 3DTP* & $422267(37.60)$ \\
1DTP & $31777(2.83)$ \\
2DTP & $136062(12.12)$ \\
1DTP+MMR & $12269(1.09)$ \\
2DTP+MMR & $70040(6.24)$ \\
3DTP+MMR & $430210(38.31)$ \\
None & $18761(1.67)$ \\
MMR & $1543(0.14)$ \\
Total & $1122929(100.00)$ \\
\hline
\end{tabular}

${ }^{*}$ Reference group in the Results section.

DTP, diphteria-tetanus-pertussis-polio-Haemophilus influenzae type b; MMR, measles-mumps-rubella. 
Table 2 Absolute number of the primary events mortality, hospitalisation for infection and onset of asthma, and for the last two outcomes, the number of competing events (deaths)

\begin{tabular}{|c|c|c|c|c|c|}
\hline \multirow[b]{3}{*}{$\begin{array}{l}\text { Vaccination } \\
\text { status }\end{array}$} & \multicolumn{5}{|c|}{ No of events by type of outcome } \\
\hline & \multirow{2}{*}{$\begin{array}{l}\text { Mortality } \\
\text { Primary event } \\
\text { (no competing event) }\end{array}$} & \multicolumn{2}{|c|}{ Hospitalisation for infection } & \multicolumn{2}{|c|}{ Onset of asthma } \\
\hline & & Primary event & $\begin{array}{l}\text { Competing event } \\
\text { (deaths) }\end{array}$ & Primary event & $\begin{array}{l}\text { Competing event } \\
\text { (deaths) }\end{array}$ \\
\hline 3DTP* & 93 & 21562 & 42 & 14237 & 78 \\
\hline 1DTP & 39 & 2527 & 13 & 1446 & 27 \\
\hline 2DTP & 68 & 8520 & 23 & 5335 & 58 \\
\hline 1DTP+MMR & 9 & 3613 & 6 & 2254 & 8 \\
\hline 2DTP+MMR & 65 & 19816 & 24 & 12582 & 61 \\
\hline 3DTP+MMR & 304 & 114648 & 142 & 73534 & 257 \\
\hline None & 29 & 1819 & 10 & 809 & 25 \\
\hline MMR & $<5$ & 534 & $<5$ & 301 & $<5$ \\
\hline Total† & $607-612$ & 173039 & $260-265$ & 110498 & $514-519$ \\
\hline
\end{tabular}

The numbers are shown by vaccination status and in total.

${ }^{*}$ Reference group in the Results section.

†The number of competing events was lower than the death total, since some deaths took place after the primary event.

DTP, diphteria-tetanus-pertussis-polio-Haemophilus influenzae type b; MMR, measles-mumps-rubella.

hand, the reference group of children with 3 DTPs and no MMR represented 422267 children or $37.60 \%$ of the children in the cohort. This is important to bear in mind before drawing conclusions from the comparisons between the different vaccination groups. As mentioned in the Methods section, multiple DTP administrations to the same child within the same week were considered as one vaccination.

In table 2, the number of events is presented for each of the three outcomes. The total number of events during the study period are presented along with the subtotals for each of the vaccination groups. The two non-mortality outcomes are prone to the competing risk of death and as such the number of competing events is also presented.

\section{Effect of vaccination on mortality and morbidity}

The analyses examined the association between vaccination group and mortality, hospitalisation for infection and asthma, and the result of confounder adjustment (table 3 ). The aHRs are presented below with 95\% CIs in parenthesis (the unadjusted HRs can also be found in table 3). No serious violations of the proportional hazards assumption related to the vaccination status exposure were detected.

Mortality

Children with 1 or 2 DTP only were found to have higher hazards of dying than that of the reference group of children with 3 DTP: aHR=1.96 (95\% CI: 1.34 to 2.89) and aHR $=1.55$ (95\% CI: 1.14 to 2.13).

Table 3 Crude and adjusted HRs for each outcome. The estimated parameters were HRs. The reference group was the group of children with 3DTP, but no MMR

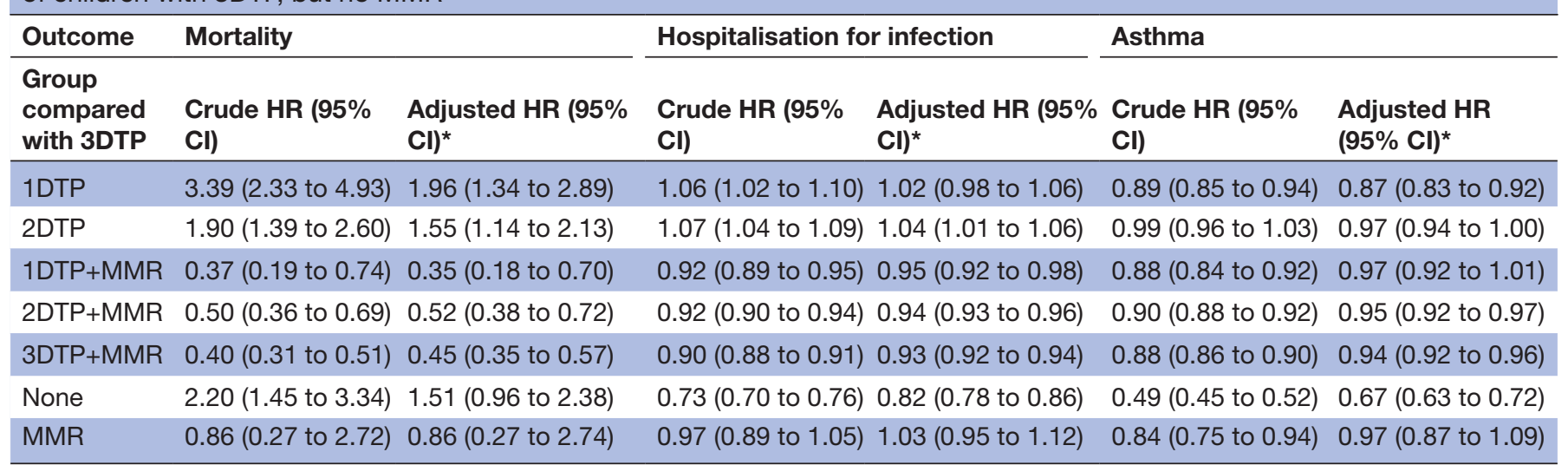

${ }^{*}$ The parameter estimates corresponding to the confounders are presented in in online supplementary table S7.

DTP, diphteria-tetanus-pertussis-polio-Haemophilus influenzae type b; MMR, measles-mumps-rubella. 
Children with 3 DTP+MMR were found to have a lower hazard of dying compared with that of the 3 DTP group: $\mathrm{aHR}=0.45$ (95\% CI: 0.35 to 0.57 ). Based on the CIs, the hazards of children with 1, 2 or $3 \mathrm{DTP}+\mathrm{MMR}$ were similar.

\section{Hospitalisation for infection}

Children with 1 or 2 DTP only were found to have similar hazards of being hospitalised for infection compared with the reference group of children with $3 \mathrm{DTP}$ : $\mathrm{aHR}=1.02$ (95\% CI: 0.98 to 1.06 ) and aHR=1.04 (95\% CI: 1.01 to 1.06). Based on the CIs, the hazard in the 1 DTP group was not different from that in the 3 DTP groups.

Children with 3 DTP+MMR were found to have a slightly lower hazard of being hospitalised for infection than that of the 3 DTP group: aHR=0.93 (95\% CI: 0.91 to 0.94) . Based on the CIs, the hazards of children with 1, 2 or $3 \mathrm{DTP}+\mathrm{MMR}$ were similar.

\section{Asthma}

Children with 1 or 2 DTP were found to have lower hazards of developing asthma than that of the reference group of children with 3 DTP: aHR $=0.87$ (95\% CI: 0.83 to $0.92)$ and $\mathrm{aHR}=0.97$ (95\% CI: 0.94 to 1.00$)$. Based on the CIs, the hazard in the 2 DTP group was not significantly different from that in the 3 DTP group.

Children with 3 DTP+MMR were found to have a slightly lower hazard than that of the 3 DTP group: aHR $=0.94$ (95\% CI: 0.92 to 0.96 ). Based on the CIs, the hazards of children with 1, 2 or 3 DTP+MMR were similar.

\section{The remaining vaccination groups: no vaccines and MMR only}

Two of the smallest vaccination groups at 16 months of age were the group of children without DTP or MMR (labelled 'None') and the group of children with MMR only. At baseline, the two groups represented $1.67 \%$ and $0.14 \%$ of the cohort, respectively, and were not of primary interest. The children with no vaccinations had a lower estimated hazard of hospitalisation for infection and onset of asthma. The characteristics of this group were peculiar. For example, $98.09 \%$ of the children with a missing value of the ethnicity variable (462 of 471) had no vaccinations at 16 months age, which was extreme bearing in mind that these children only constituted $1.67 \%$ of the cohort. Information available on request and is given in online supplementary tables S5 and S6.

\section{The result of adjustment}

The largest differences between the crude HR and aHRs were found in the mortality outcome. In particular, the mortality estimates corresponding to the 1 and 2 DTP groups were largely reduced after adjustment. However, the HR corresponding to the group of children with no vaccinations (the None group) also changed somewhat after adjustment in the outcomes hospitalisation for infection and onset of asthma. This suggests that some of the peculiarity in the composition of the None-group was captured by the differences in the observed confounder distributions.

\section{Alternative parametrisation}

The results presented in table 3 are based on analysis with one exposure variable with eight levels— the four times two combinations of DTP and MMR. Alternatively one could define two separate variables with four levels, DTP $\in\{0,1,2,3\}$, respectively two levels, MMR $\in\{0,1\}$, and study their interaction. The result of such an analysis is presented in online supplementary table S8.

\section{Absolute risks}

The absolute risk is the probability of experiencing the outcome. The vaccination-specific absolute risks of experiencing the main outcomes were calculated from $16,20,24,36$ and 48 months until 5 years of age, and as explained in the Methods section, these are the time points at which the vaccination status exposure is re-evaluated. The results from the landmark time points of 16 months and 36 months (3 years) of age are presented in table 4 . Here the relative risks are ratios between two absolute risks. The number of deaths diminished toward the end of follow-up making the mortality estimates uncertain. The landmark results from 20, 24 and 48 months of age can be found in the online supplementary table S9, where the vaccination-specific results corresponding to the two groups without DTP (None and MMR) are also presented (online supplementary table S10).

Based on the $95 \%$ CIs, the mortality risks of the 3DTP and 3DTP+MMR groups appear similar. This differs from the result of the cause-specific hazards analysis. One notable difference between the interpretations is that the landmark analysis from 16 months of age does not consider children who are vaccinated with MMR more than 1 month later than scheduled. For all vaccination groups at all landmark times, the absolute risk of dying was negligible. Otherwise the relative risks are close to 1 indicating that for these outcomes the different vaccination regimens had no clinically important impact on the risks: no matter the vaccination status at 16 months of age, the risk of experiencing a hospitalisation for infection before 5 years of age was around $16 \%$, and the risk of asthma was around $10 \%$. For the event-free children surviving up to 3 years of age, the risks were around $5 \%$ for hospitalisation for infection and around $2 \%$ for asthma.

\section{Negative control outcome: examining residual confounding}

The absolute risks estimated using the g-formula represent average causal effects if there are no unmeasured confounders. To investigate this assumption, the negative control outcome 'hospitalisations related to accidents' was analysed and the results of the cause-specific Cox regression model with this outcome are presented in table 5 .

Children with 1 or 2 DTP only were found to have similar hazards of being hospitalised due to accidents than that of the reference group of children with 3 DTP: aHR=1.09 (95\% CI: 1.00 to 1.19$)$ and $\mathrm{aHR}=1.05$ (95\% CI: 0.99 to 1.11$)$. 
Table 4 For each of the two outcomes: hospitalisation for infection (middle part of table) and asthma (lower part of table): 5 years of age absolute and relative risks (3DTP as reference) are presented by vaccination status (apart from the two groups without DTP) at landmark times 16 and 36 months of age (with 95\% Wald Cls). For the mortality outcome (upper part of table), only relative risks are presented (all absolute mortality risks <0.001)

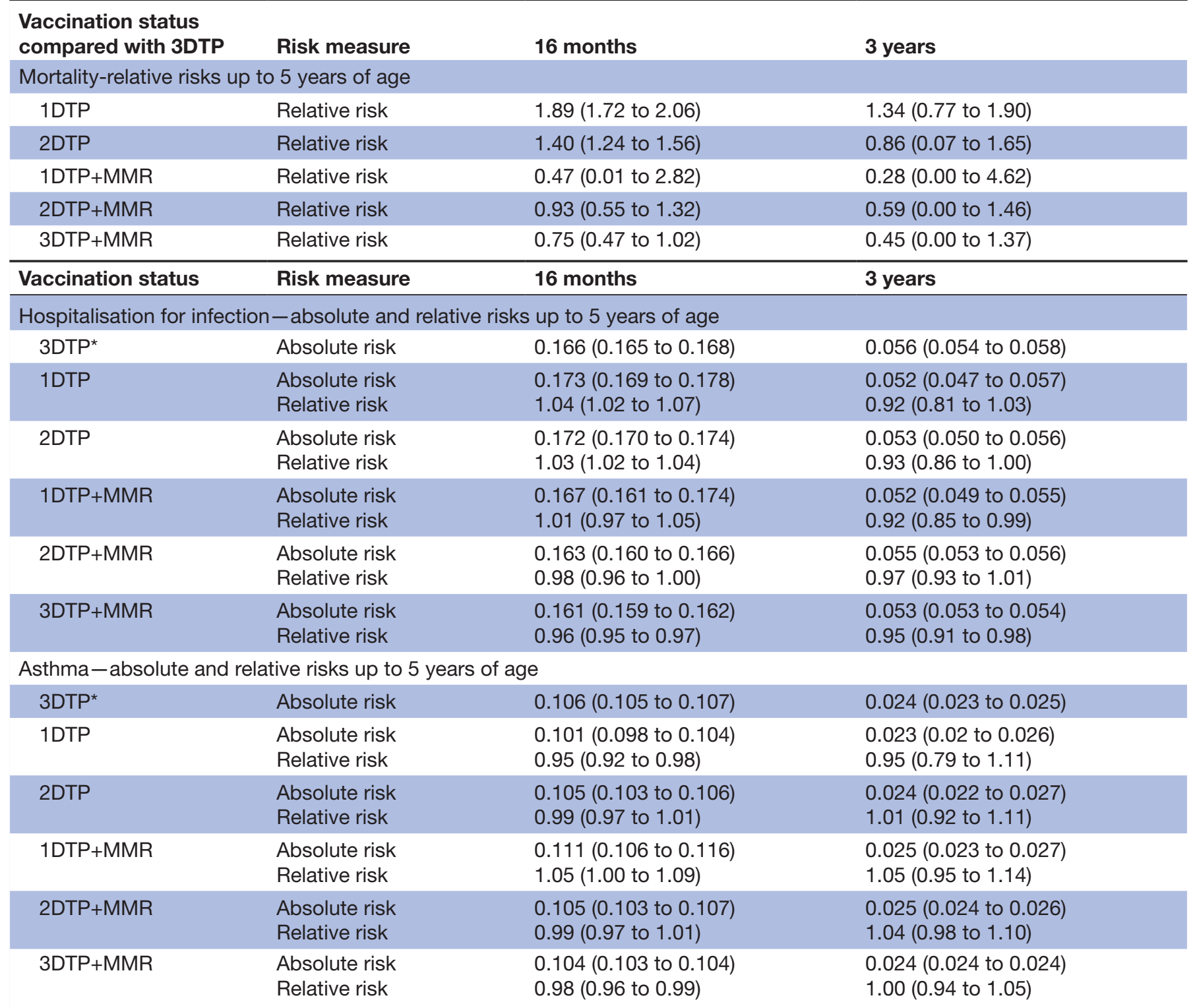

*The reference group is 3DTP.

DTP, diphteria-tetanus-pertussis-polio-Haemophilus influenzae type b; MMR, measles-mumps-rubella.

Children with 3 DTP+MMR were found to have a lower hazard of being hospitalised due to accidents compared with that of the 3 DTP group: aHR=0.83 (95\% CI: 0.80 to $0.85)$. The hazards of children with 1,2 or $3 \mathrm{DTP}+\mathrm{MMR}$ were similar based on the CIs.

Thus, the results of the negative control outcome analysis suggest that the mechanisms explaining exposure to MMR remain partly uncontrolled for.

\section{DISCUSSION}

\section{Findings}

This national cohort study of more than 1.12 million Danish children aimed at estimating the association between early childhood vaccination and subsequent mortality and morbidity. The study found an association between MMR and reduced mortality, but no detrimental effect of DTP. However, the study also found an association between MMR and hospitalisation for accidents indicating residual confounding, so-called 'healthy vaccinee bias'.

Many children did not receive MMR by 16 months of age. In Denmark, it is well-known than parents tend to forget/ postpone the MMR vaccine scheduled at 15 months of age to a larger degree than it is the case for the 3 DTP vaccines at 3, 5 and 12 months of age. Usually parental leave lasts 12 months in Denmark covering the timing of the DTP vaccines, but not the MMR. When children enter daycare, their risk of infection and fever increases which 
Table 5 Crude and adjusted HRs for the negative control outcome. The estimated parameters were HRs. The reference group was the group of children with 3DTP, but no MMR

\begin{tabular}{lll}
\hline $\begin{array}{lll}\text { Outcome } \\
\text { Group compared } \\
\text { with 3DTP }\end{array}$ & \multicolumn{2}{l}{ Hospitalisation due to accidents } \\
\cline { 2 - 3 } 1DTP & Crude HR $(95 \%$ Cl) & Adjusted HR $(95 \%$ Cl) \\
\hline 2DTP & $1.12(1.03$ to 1.22$)$ & $1.09(1.00$ to 1.19$)$ \\
1DTP+MMR & $1.07(1.01$ to 1.13$)$ & $1.05(0.99$ to 1.11$)$ \\
\hline 2DTP+MMR & $0.83(0.77$ to 0.89$)$ & $0.84(0.79$ to 0.91$)$ \\
\hline 3DTP+MMR & $0.83(0.79$ to 0.86$)$ & $0.84(0.80$ to 0.87$)$ \\
\hline None & $0.81(0.78$ to 0.83$)$ & $0.83(0.80$ to 0.85$)$ \\
\hline MMR & $0.83(0.76$ to 0.91$)$ & $0.92(0.84$ to 1.01$)$ \\
\hline
\end{tabular}

DTP, diphteria-tetanus-pertussis-polio-Haemophilus influenzae type b; MMR, measles-mumps-rubella.

again tends to postpone the date of vaccination. In the landmark approaches any postponed uptake before the given landmark time point was accounted for.

\section{Strengths and weaknesses}

The strengths of the present study include the large sample size, the national cohort study design, the inclusion of all combinations of vaccines, estimation of absolute risks, the flexibility of the time-dependent vaccination exposure status and the negative control outcome of hospitalisation due to accidents which cannot readily be pathogenetically related to the vaccine exposure. In the present study, we elaborated on the analytical strategy of a recent study from the Netherlands. ${ }^{7}$ Here the authors claimed to have found that the decreased risk of adverse health outcomes was associated with receipt of any additional vaccine and not specifically the live-attenuated MMR vaccine. However, in that study only children with at least 3 or 4 DTP vaccinations were included in the analysis, while the present study extensively examined all combinations of early childhood vaccines administered according to the Danish vaccination programme. We broadened the comparison groups by not restricting the population to children who received at least 3 DTP vaccinations. ${ }^{7}$ This enabled the observation of decreased mortality with an increased number of DTP vaccines in the absence of MMR.

One weakness of all observational studies is the possibility of unmeasured confounding. It is not known whether further adjustment for relevant confounders would change the estimated HRs, but the association between MMR and hospitalisation due to accidents indicates a problem with the confounder control. The unmeasured confounders could include medication administered in hospital which is not measurable on an individual level in the Danish national health registries, immunodeficiencies and other severe chronic diseases not captured by our definition of chronic disease, and other measures of somatic frailty which are hard to identify. In addition, several social aspects and family resources which are hard to capture in the national registries could influence the results.

Other weaknesses include potential misclassifications of exposure or outcome status and other variables used in the analyses. Regarding misclassification of the vaccination status, the misclassification in reimbursement-based register data is likely to be minimal due to financial incentives for the general practitioners who administer the vaccines, and whenever present any misclassification is most likely non-differential, that is, individuals were misclassified with equal probability across all groups in the study. Regarding misclassification of outcomes: mortality information is reliable, whereas hospitalisations and asthma can be prone to misclassification. The former only to a small extent since it is in the interest of the hospital department that the diagnoses are coded correctly to obtain reimbursement. Asthma is not ascertained without error as seen in our previous validation study of the algorithm defining asthma.

\section{Note on the children with no vaccines}

The children without any vaccines at 16 months of age constitute a small subgroup (1.67\%) which is not representative of the general population. In fact, a lot of these ill children might have been vaccinated on the hospital and not by the general practitioner-and this study is based on information from the latter. The very fact that these children were possibly vaccinated at the hospital suggest that they represent a very ill subgroup. The frequency of missing data in this among these children (online supplementary table S5) can be due to their complicated births: typically, the midwife ascertains the information after birth, but this procedure has low priority in these few, severe cases. These children barely use the remaining health system and have few incident hospitalisations for infections since they might already be hospitalised, or they are hospitalised for even more severe reasons, and thus having no vaccines appears beneficial when the outcome is ascertained by register-based non-mortality outcomes. But they die a lot earlier than the remaining population.

\section{Comparison with other studies}

Since the negative control outcome, as already mentioned, points toward residual confounding, our study also suggests that residual confounding may have been present in previous Danish register-based studies investigating the impact of MMR on the morbidity outcomes respiratory syncytial virus and hospitalisation for infection until 2 years of age ${ }^{34}$ and asthma-like disease until 5 years of age. ${ }^{5}$ A previous study found no association between MMR and the rate of emergency room visits. ${ }^{6}$

The estimated HR for mortality after administration of MMR seems large. However, effect sizes of this magnitude are in line with prior estimates ${ }^{2}$; based on this it is not uncommon to estimate halved mortality hazards. As for all estimates in the present study and prior studies, 
this HR may be prone to biases from sources similar to the ones discussed under the section Note on children with no vaccines above: some children who do not receive MMR suffer from disease such as immunodeficiencies and socioeconomic disadvantages which again affect the analysed outcomes.

\section{Meaning of study, explanations and implications}

The study points at reduced mortality after MMR vaccination, but any additional DTP vaccination was not associated with increased mortality; in fact, any additional vaccination with DTP even seemed to be associated with reduced mortality in the group of children without MMR. No clinically important differences between vaccination groups were found for the outcomes hospitalisation for infection and asthma even though an increased hazard of asthma with the number of DTP vaccinations was estimated.

\section{Unanswered questions and future research}

Based on the results, we raise the question if the lower risk of mortality in children with MMR may to some degree be explained by unobserved confounding by, for example, contraindication of live-attenuated vaccines in immunocompromised individuals with a very high risk of adverse health events which was not appropriately adjusted for even after the inclusion of the 12 relevant confounders. Therefore, non-specific effects of vaccines are best examined in randomised trials.

Contributors All authors conceived the study idea. AJ and LGS defined and obtained the register data. AJ carried out the data management and statistical analyses. LGS and PKA supervised the data management and statistical analyses. AJ drafted the manuscript. All authors revised the manuscript critically for important intellectual content. All authors read and approved the final manuscript.

Funding This study was financed by The Lundbeck Foundation (grant no R208-2015-3384).

\section{Competing interests None declared.}

Patient consent for publication Not required.

Provenance and peer review Not commissioned; externally peer reviewed.

Data availability statement Data may be obtained from a third party and are not publicly available. The use of and access to all data were secured within the servers of Statistics Denmark. All data were fully pseudonymised.

Open access This is an open access article distributed in accordance with the Creative Commons Attribution Non Commercial (CC BY-NC 4.0) license, which permits others to distribute, remix, adapt, build upon this work non-commercially, and license their derivative works on different terms, provided the original work is properly cited, appropriate credit is given, any changes made indicated, and the use is non-commercial. See: http://creativecommons.org/licenses/by-nc/4.0/.

\section{REFERENCES}

1. Yung CF. Non-Specific effects of childhood vaccines. BMJ 2016;355:i5434-3.

2. Higgins JPT, Soares-Weiser K, López-López JA, et al. Association of BCG, DTP, and measles containing vaccines with childhood mortality: systematic review. BMJ 2016;355.

3. Sørup S, Benn CS, Stensballe LG, et al. Measles-mumps-rubella vaccination and respiratory syncytial virus-associated Hospital contact. Vaccine 2015;33:237-45.

4. Sørup S, Benn CS, Poulsen A, et al. Live vaccine against measles, mumps, and rubella and the risk of hospital admissions for nontargeted infections. JAMA 2014;311:826-35.
5. Hviid A, Melbye M. Measles-Mumps-Rubella vaccination and asthma-like disease in early childhood. Am J Epidemiol 2008;168:1277-83.

6. Sørup S, Benn CS, Poulsen A, et al. Simultaneous vaccination with MMR and DTaP-IPV-Hib and rate of hospital admissions with any infections: a nationwide register based cohort study. Vaccine 2016;34:6172-80.

7. Tielemans SMAJ, de Melker HE, Hahné SJM, et al. Non-Specific effects of measles, mumps, and rubella (MMR) vaccination in high income setting: population based cohort study in the Netherlands. BMJ 2017;358.

8. Donzelli A. Influenza vaccinations for all pregnant women? better evidence is needed. Int J Environ Res Public Health 2018;15:2034.

9. McElhaney JE, Andrew MK, McNeil SA. Estimating influenza vaccine effectiveness: evolution of methods to better understand effects of confounding in older adults. Vaccine 2017;35:6269-74.

10. Remschmidt C, Wichmann O, Harder T. Frequency and impact of confounding by indication and healthy vaccinee bias in observational studies assessing influenza vaccine effectiveness: a systematic review. BMC Infect Dis 2015;15.

11. Jackson LA, Nelson JC, Benson P, et al. Functional status is a confounder of the association of influenza vaccine and risk of all cause mortality in seniors. Int J Epidemiol 2006;35:345-52.

12. MMRVAXPRO. Summary of product characteristics, 2015. Available: http://www.ema.europa.eu/docs/en_GB/document_library/EPAR_-Product_Information/human/000604/WC500030170.pdf

13. European Medicines Agency. MMRVAXPRO, 2015. Available: http://www.ema.europa.eu/ema/index.jsp?curl=pages/medicines/ human/medicines/000604/human_med_000907.jsp\&mid= WC0b01ac058001d124

14. Danish Medicines Agency, Pro.medicine, 2018. Available: DiTeKiPol/ Act-Hib

15. Christenfeld NJS, Sloan RP, Carroll D, et al. Risk factors, confounding, and the illusion of statistical control. Psychosom Med 2004;66:868-75.

16. Stensballe LG, Sørup S, Aaby P, et al. Bcg vaccination at birth and early childhood hospitalisation: a randomised clinical multicentre trial. Arch Dis Child 2017;102:224-31.

17. Thygesen LC, Daasnes C, Thaulow I, et al. Introduction to Danish (nationwide) registers on health and social issues: structure, access, legislation, and archiving. Scand J Public Health 2011;39(7 suppl):12-16.

18. Danmarks Statistik. Register- OG variabeloversigter, 2016. Available: http://www.dst.dk/da/TilSalg/Forskningsservice/Data/Register_ Variabeloversigter.aspx

19. Pedersen CB, Gøtzsche H, Møller JO, et al. The Danish civil registration system. A cohort of eight million persons. Dan Med Bull 2006;53:441-9.

20. Schmidt M, Schmidt SAJ, Sandegaard JL, et al. The Danish national patient registry: a review of content, data quality, and research potential. Clin Epidemiol 2015:449-90.

21. Wallach Kildemoes H, Toft Sørensen H, Hallas J. The Danish national prescription registry. Scand J Public Health 2011;39(7 suppl):38-41.

22. Bliddal $M$, Broe $A$, Pottegård $A$, et al. The Danish medical birth register. Eur J Epidemiol 2018;33:27-36.

23. Sahl Andersen J, De Fine Olivarius N, Krasnik A. The Danish National health service register. Scand J Public Health 2011;39(7_ suppl):34-7.

24. Danish Health Authority. The Danish childhood vaccination programme, 2016. Available: https://sundhedsstyrelsen.dk/ en/disease-and-treatment/vaccination/childhood-vaccinationprogramme

25. Sørup S, Villumsen M, Ravn $\mathrm{H}$, et al. Smallpox vaccination and all-cause infectious disease hospitalization: a Danish register-based cohort study. Int J Epidemiol 2011;40:955-63.

26. Rieckmann A, Villumsen M, Sørup S, et al. Vaccinations against smallpox and tuberculosis are associated with better long-term survival: a Danish case-cohort study 1971-2010. Int J Epidemiol 2017;46:695-705.

27. Henriksen L, Simonsen J, Haerskjold A, et al. Incidence rates of atopic dermatitis, asthma, and allergic rhinoconjunctivitis in Danish and Swedish children. J Allergy Clin Immunol 2015;136:360-6.

28. Stensballe LG, Klansø L, Jensen A, et al. The validity of register data to identify children with atopic dermatitis, asthma or allergic rhinoconjunctivitis. Pediatr Allergy Immunol 2017;28:535-42.

29. VanderWeele TJ, Shpitser I. A new criterion for Confounder selection. Biometrics 2011;67:1406-13.

30. Talge NM, Mudd LM, Sikorskii A, et al. United States birth weight reference corrected for implausible gestational age estimates. Pediatrics 2014;133:844-53. 
31. Kristensen $\mathrm{K}$, Hjuler T, Ravn $\mathrm{H}$, et al. Chronic diseases, chromosomal abnormalities, and congenital malformations as risk factors for respiratory syncytial virus hospitalization: a population-based cohort study. Clin Infect Dis 2012;54:810-7.

32. Ozenne B, Sørensen Anne,Lyngholm, Scheike T, et al. riskRegression: predicting the risk of an event using COX regression models. $R$ J 2017:9:440-60.

33. Naimi Al, Cole SR, Kennedy EH. An introduction to G methods. Int J Epidemiol 2017;46:756-62.
34. Hernán MA, Robins JM. Estimating causal effects from epidemiological data. J Epidemiol Community Health 2006;60:578-86.

35. van Houwelingen $\mathrm{HC}$, Putter $\mathrm{H}$. Dynamic predicting by landmarking as an alternative for multi-state modeling: an application to acute lymphoid leukemia data. Lifetime Data Anal 2008;14:447-63.

36. Lipsitch M, Tchetgen Tchetgen E, Cohen T. Negative controls: tool for detecting confounding and bias in observational studies. Epidemiology 2010;21:383-8. 